\title{
current events
}

This section carries events of interest to the synchrotron radiation community. Works intended for this section should be sent direct to the Current-Events Editor (s.hasnain@dl.ac.uk).

\section{Praveen Chaudhari takes the helm at Brookhaven National Laboratory}

Dr Praveen Chaudhari became the Director of Brookhaven National Laboratory, which hosts the National Synchrotron Light Source (NSLS), from 1 April 2003. Prior to this he spent 36 years at IBM where he rose from a member of the research team to the Vice President of Science in 1982. He is well known to the synchrotron radiation community through his work on condensed matter physics. Dr Chaudhari gained his bachelors degree from the Indian Institute of Technology, Kharagpur, and earned his doctorate in 1966 from the Massachusetts Institute of Technology in physical metallurgy. His recent research interests have been in nanoscience and superconductivity.

The science programs flourished during Chaudhari's management tenure in the IBM Research Division. The work carried out became the basis of the USD 2-billion-a-year optical-disk industry and IBM scientists captured Nobel Prizes in physics for two consecutive years, in 1986 for developing the scanning tunneling microscope, and in 1987 for discovering high-temperature superconductivity in a new class of materials.

Chaudhari was executive secretary of President Reagan's Advisory Council on Superconductivity (1988). He was a member of the National Commission on Superconductivity, which reported its findings to President Bush (1989). In 1988, Chaudhari reported on science and technology to Prime Minister Rajiv Gandhi of India; in 1993, at the request of the Indian Minister for Sciences and Technology, he led an IBM group to evaluate India's parallel computer activities. He is a fellow of the American Academy of Arts and Sciences, a fellow of the American Physical Society, a member of the National Academy of Engineering and an Associate Fellow of the Third World Academy of Sciences.

\section{Funding for first phase of 4 GLS announced}

On 2 April, the UK Government announced the funding (GBP 11.5 million) for the first phase of the fourth-generation light source (4GLS). The funding supports the first phase of the project, a three-year research, development and design study to address the technical challenges and establish the technical know-how needed to build this innovative facility. This will also provide Daresbury with an important resource for research in other areas of accelerator science.

Lord Sainsbury, the UK's Minister for Science, said, "This threeyear study into the technology behind 4GLS is an important step towards placing Daresbury at the cutting edge of accelerator science. 4GLS would provide scientists with a first-class facility to conduct vital experiments in many disciplines. Its potential capability is unique in the world, and its capacity to combine a wide range of experiments would establish the UK as a major international player in this technology."

\section{Diamond construction commences}

Construction of the Diamond Light Source passed a major milestone on 12 March when Dr John Taylor, Director General of Research Councils in the United Kingdom, cut the first turf from its site at the Rutherford Appleton Laboratory in Oxfordshire. Funded jointly by the UK government, through CCLRC, and the Wellcome Trust, the GBP 235 million project is planned to begin operations in January 2007. Dr Taylor commented that this is the largest science facility development in the UK for 30 years and demonstrates its commitment to providing scientists with world-class research opportunities.

The 24-cell storage ring will have a circumference of $562 \mathrm{~m}$ and operate at $3.0 \mathrm{GeV}$ with superconducting RF cavities. Seven beamlines will be available at the start of operations for users in physical and life sciences. These include three beamlines for protein crystallography and one for microfocus XAFS. Further beamlines will be added at a rate of four per year to a complement of 21. The full complement of beamlines is expected to be ready for users in 2012.

Diamond Light Source (DLS) Ltd was formed in March 2002 with a board under the chairmanship of Sir David Cooksey. The Diamond CEO, Professor Gerd Materlik, expressed his determination to confront the technical challenges for stability and precision and to create a new focal point for science in the UK. CCLRC, which is also responsible for running the Daresbury Laboratory, is the major shareholder in DLS. Its CE, Professor John Wood, said that he was delighted to see that the construction of Diamond had now started. CCLRC were now looking to the future with exciting developments at Daresbury concerning the fourth-generation light-source (4GLS) project. Meanwhile the operation of the current SRS will be focused on, ensuring that it can deliver world-leading science on its beamlines for the benefit of all users.

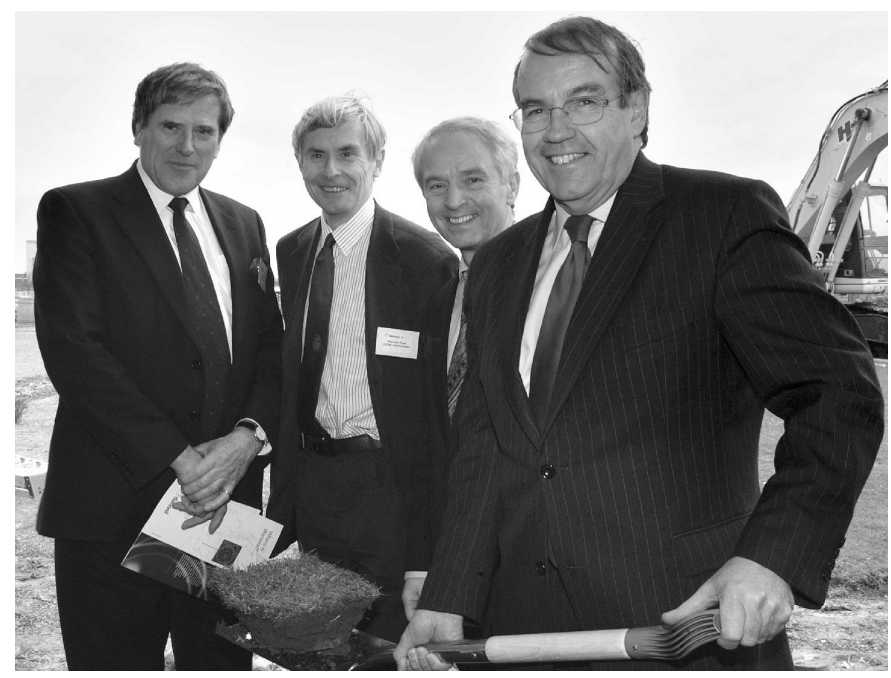

Dr John Taylor, Director General of Research Councils in the United Kingdom, cuts the first turf from the Diamond site at the Rutherford Appleton Laboratory. Also pictured are, from left to right, Stuart Doughty, Chief Executive of Costain who are responsible for the building construction, John Wood and Gerd Materlik. 
USA prepares a 20-year roadmap for basic energy sciences facilities

Ray Orbach, the Director of the Office of Science at the Department of Energy, received a detailed report from a committee chaired by Geraldine Richmond (University of Oregon) and Sunil Sinha (University of California, San Diego). The committee primarily looked at the synchrotron radiation and neutron provision for the USA. For the Journal of Synchrotron Radiation readership, we summarize the main aspects of recommendations affecting the synchrotron radiation community.

The committee recognized the importance of four DOE light sources for the country's scientific and technological enterprise and stressed that, in order to continue producing cutting-edge science, upgrades at these facilities are needed in the near future for them to remain at the forefront of light-source activities. DOE should aggressively pursue a light source facilities initiative that involves a coordinated effort among the four light sources, namely:

(i) The National Synchrotron Light Source (NSLS) and the Office of Basic Energy Sciences (BES) to formulate a plan for construction of a third-generation ring at NSLS instead of the current very ambitious plan for NSLS II which is a fourth-generation source. The committee commented that the scope of the current NSLS II proposal is too broad and does not take account of DOE's national facilities portfolio.

(ii) The Advanced Photon Source (APS): phase I and II upgrades were strongly supported, namely (a) to complete the installation of the remaining beamlines and to optimize the earlier beamlines, and (b) to optimize the source by means of innovative insertion devices and accelerator development. (iii) The Advanced Light Source (ALS): support of their upgrade proposal to go to full energy injection and higher current, as well as to replace obsolescent insertion devices (IDs) and beamlines with stateof-the-art IDs and new beamlines.

(iv) Stanford Synchrotron Radiation Laboratory (SSRL) is currently undergoing an upgrade that will make it a third-generation $\mathrm{X}$-ray source.

Among the new facilities in the immediate future, one proposal that ranked very highly in both quality of proposed science and facility readiness was the Linear Coherent Light Source (LCLS). This free-electron laser (FEL) facility is essential for exploring future science using intense femtosecond coherent X-ray beams. The knowledge gained from operation of this facility is critical for providing preliminary assessments of many science drivers for these new intense coherent fast X-ray sources and providing critical guidance to the planning and ultimate success of several future proposed X-ray FEL (XFEL) sources that offer exciting new prospects for future scientific endeavors in BES. The BESAC subcommittee enthusiastically support this project.

The committee commented that if predicted successes of LCLS are realised, the community will demand a full-scale, state-of-the-art, fully coherent, superbright, multiplexed, diffraction-limited facility, the so called 'Greenfield' XFEL. The committee feels that the appropriate research and development that could enable this facility must go forward. Critical areas that need improvement include gun technology, detector optimization and electron beam stability, as well as continued advances in the scientific case. Within five years, experience with XFEL science and technology should mature to the point that a decision can be made on the scope of any future Greenfield XFEL facility. 